\title{
Transfer Management of Healthcare Archive Samples for Oncological Clinical Trial by the Biobank Platform
}

Martínez B. ${ }^{1}$, Ferro P. ${ }^{1}$, Jiménez A. ${ }^{1}$, Fernández M.I. ${ }^{1}$, Díaz T. ${ }^{1}$, Martín I. ${ }^{1}$ Acosta-Andrade C. ${ }^{1}$ and Hortas M.L. ${ }^{2}$

${ }^{1}$ Andalusian Public Health System Biobank, Instituto de Investigación Biomédica de Málaga (IBIMA), Sanitary Distric of Málaga, University of Málaga.

${ }^{2}$ Costa del Sol Sanitary Agency, Sanitary District of Málaga.

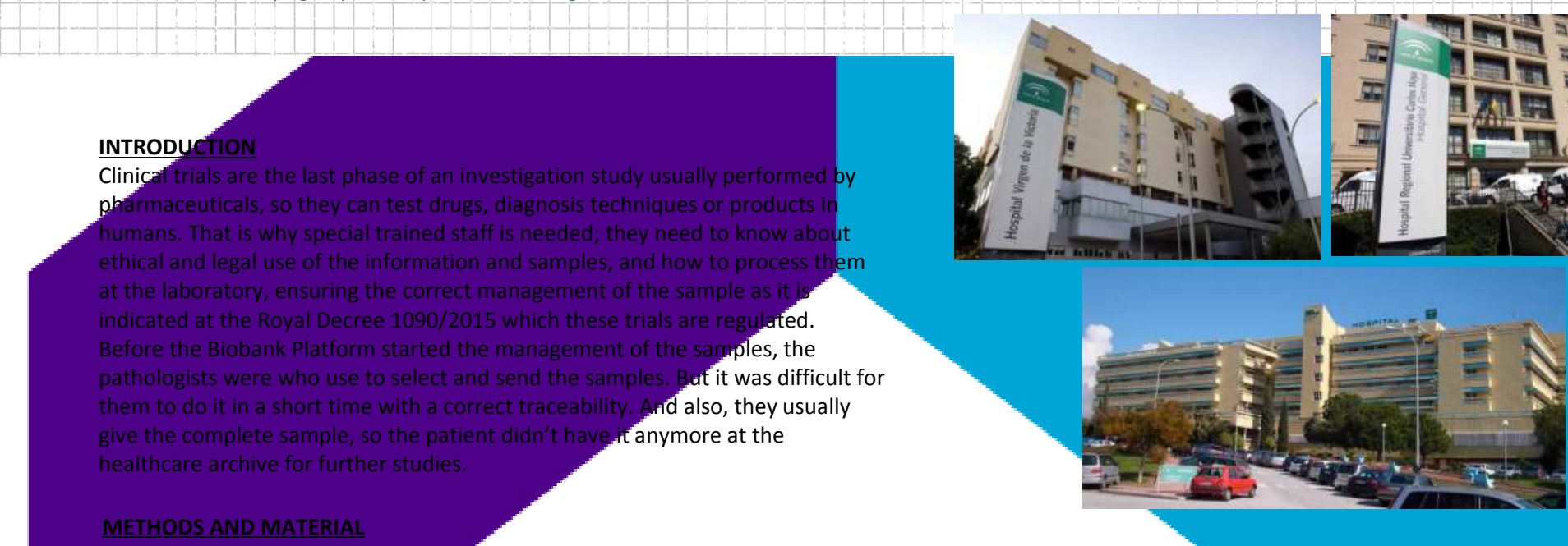

patients privacy and the correct process of the samples if it will be needed, sending just the minimum amount of quirements, at any of the Malaga hospitals: Virgen de la Victoria Hospital; Malaga's Regional Hospital, which del Sol Hospital, in Marbella.

ee, since the sample is obtained until the delivery manager sends it to the pertinent laboratory (study coordinator). the material in case the patient or the pathologist need it again, so we can go back and know where it is. To do so in case the complete block is given, and recorded at the Pathological Anatomy database. In any case, the block or Biobank database, so there's a triple check system to record all the needed information.

me (Figure 1), which is something very important because the patient is waiting the results of the laboratory to

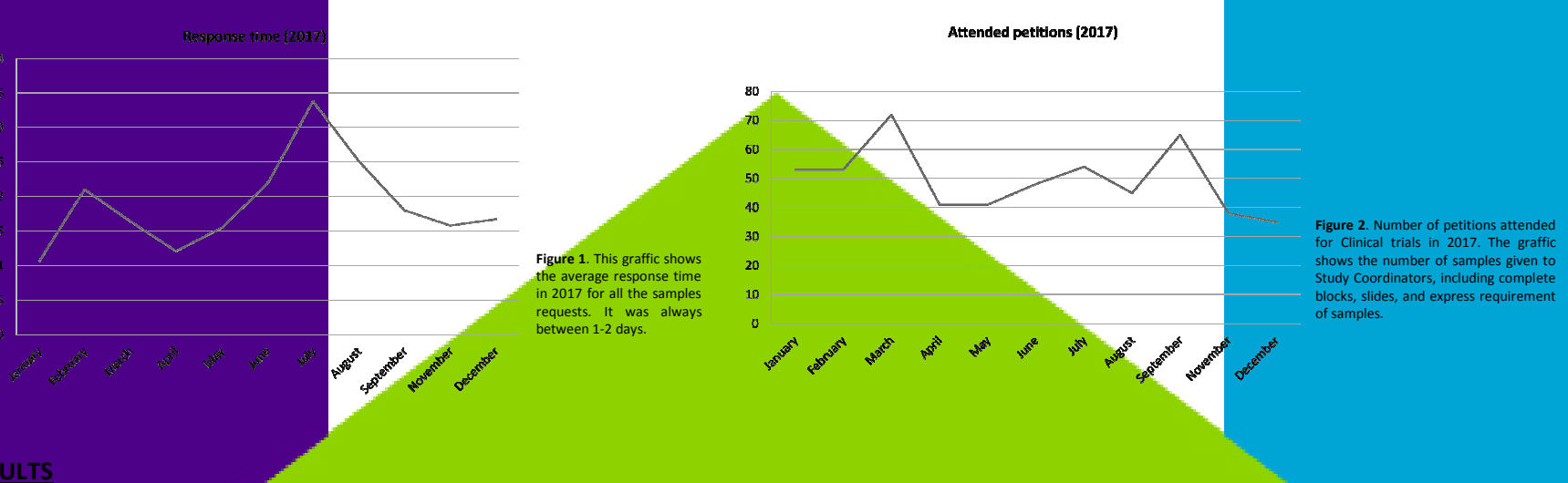

rinator, pathologist and Biobank), ensuring the tracking of the sample following the guidelines of the current legislation increase the number of petitions for Clinical trials (Figure 2).

\section{CONCLUSION}

Biobank Platform has openned an effective circuit to transfer and/or processing the healthcare archive samples from the Pathological Anatomy centres in Málaga Hospitals, to the oncological clinical trials that are being undertaking in this province.

\section{ACKNOWLEDGMENTS}

This work was carried out with the financial support of the Biobank Platform PT13/0010/0006, Health Institute Carlos III (ISCIII,Madrid, Spain).
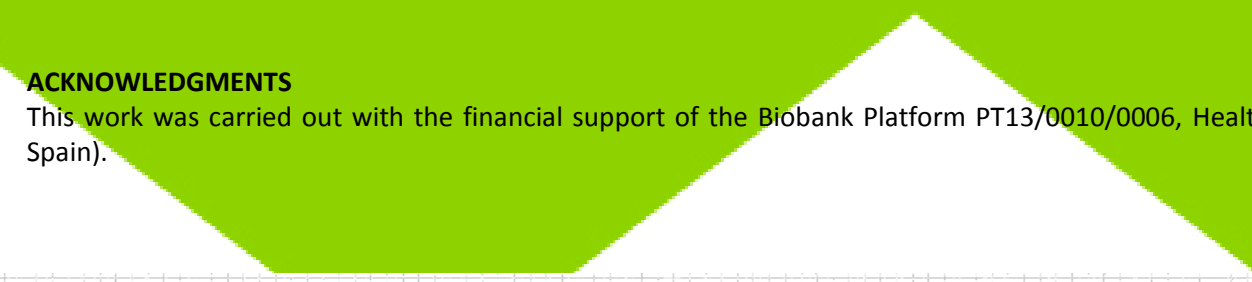